\title{
Basaloid and lobulated: Sebaceous adenoma.
}

\author{
Bajaj A* \\ A.B. Diagnostics, New Delhi, India
}

\begin{abstract}
Sebaceous adenoma is a benign epithelial neoplasm with hyperplasia of sebaceous lobules associated with expansive aggregates of basaloid germinative cells. Sebaceous adenomas usually demonstrate solitary or multiple lesions, particularly in the head and neck. Sebaceous adenomas can be associated with Muir-Torre syndrome which is a familial disorder with concomitant emergence of sebaceous neoplasm and a visceral malignancy or a concurrence of multiple keratoacanthomas and a visceral malignancy. Few to hundreds of gradually evolving, painless, yellow coloured papules or nodules with or without ulceration of the superficial epithelium are seen. Sebaceous adenomas commonly display distinct zones of clustered sebaceous lobules which are comprised of equivalent quantities of differentiated sebaceous cells and undifferentiated basaloid cells, accumulated within the papillary dermis. Mature sebaceous cells are immune reactive for Epithelial membrane antigen (EMA) and non-reactive for Carcino-embryonic antigen (CEA). Basaloid cells of sebaceous neoplasm are immune reactive for cytokeratin 7 . Sebaceous adenoma requires distinction from sebaceous hyperplasia, sebaceous carcinoma, disorders with sebaceous differentiation, clear cell variations of eccrine, melanocytic, keratinocytic and xanthomatous conditions. Sebaceous adenoma is adequately managed by a comprehensive surgical excision with consequent minimal tumour reoccurrence.
\end{abstract}

Keywords: Sebaceous adenoma, Neoplasm, Lesions, Carcino-embryonic antigen.

Accepted on August 16, 2019

\section{Introduction}

Sebaceous adenoma is an exceptional, distinctive, benign, epithelial neoplasm with sebaceous differentiation. Sebaceous adenoma was initially defined by Van Walbeek in 1949 [1]. Benign hyperplasia of sebaceous lobules accompanied by expansive aggregates of basaloid germinative cells at the tumour perimeter is characteristic of sebaceous adenoma. The condition is distinct from adenoma sebaceum, which is a cutaneous disorder described by facial angiofibromas and can be associated with tuberous sclerosis [2].

\section{Disease Characteristics}

Frequently seen on the head and neck region, sebaceous adenomas can display solitary or multiple lesions. Although uncommon in general population, sporadic variants of sebaceous adenomas or adjunctive sebaceous neoplasm can also emerge and are frequent in the head and infrequent beneath the neck. Sebaceous neoplasm appearing beneath the neck is usually indicative of Muir- Torre syndrome. Sebaceous adenomas can be associated with Muir-Torre syndrome, a condition which requires segregation in particular instances. Muir-Torre syndrome is a familial disorder which is designated by a concomitant emergence of singular or multiple sebaceous neoplasms and a visceral malignancy or a concurrence of multiple keratoacanthomas in association with a visceral malignancy. Visceral malignancies commonly visualized in Muir-Torre syndrome are colorectal carcinoma, genitourinary malignancies and breast carcinoma [2,3].

\section{Clinical Elucidation}

Sebaceous adenomas appear as gradual, painless, yellow coloured papules or nodules with or without ulceration of the superficial epithelium and are quantifiable as few to hundreds of lesions. Sebaceous adenomas appear as tan, pink or yellow nodules or papules with a maximal magnitude of 5 millimetres, commonly appear on the face or scalp and usually manifest at approximately 60 years. Sebaceous adenoma confined to the eyelid can involve glands of Zeis or Meibomian glands. Sebaceous adenomas, akin to other sebaceous proliferations, typically localize in the head and neck of elderly individuals, although adenomas can occur in several locations. Sebaceous adenoma frequently appears on the face, although the disorder can be universally detected, contingent to the localization of sebaceous glands. Majority of sporadic sebaceous adenomas arising in the absence of MuirTorre syndrome can emerge on the nose and eyelids. Distinction of nodules and papules of sebaceous adenoma is required from lesions of basal cell carcinoma on clinical examination [2,3]. 


\section{Histological Elucidation}

Sebaceous adenomas are composed of lobular proliferation of sebaceous cells with encompassing basaloid cells which can extend and be incorporated into the superimposed epidermis. Sebaceous adenomas demonstrate well defined zones of irregular, clustered sebaceous lobules within the papillary dermis. Well differentiated, lipid-rich sebaceous cells and undifferentiated basaloid cells contribute to cellular content of the neoplasm in roughly equivalent amounts. Sebaceous adenoma is a well circumscribed neoplasm, principally demonstrates an organoid and lobular configuration and frequently exhibits a contiguous or decimated superimposed squamous epithelium. Nodular and aggregated sebaceous lobules exhibit intense, darkly stained, basaloid germinative cells commingled with lightly stained, lipid-rich sebaceous cells containing intracytoplasmic vacuoles $[3,4]$. Although composed of organoid and lobular articulations, an organoid pattern can predominate in sebaceous adenoma. Expansive, undifferentiated basaloid cells are situated at the lobular margin, in contrast to lobules of sebaceous hyperplasia. Thus, enhanced quantification of undifferentiated, basaloid germinative cells is the predominant feature which distinguishes betwixt various benign sebaceous lesions such as sebaceous hyperplasia, sebaceous adenomas or sebaceous epithelioma. Sebaceous adenomas characteristically display a variable expansion of basaloid cells within increased cellular layers, which exceeds the regular dual cell layer defining normal sebaceous glands and lobules of sebaceous hyperplasia. However, the basaloid cell component remains beneath $<50 \%$ of tumour cell volume. Basaloid cell content of an estimated greater than half $(>50 \%)$ is enunciated in sebaceous epithelioma. Sebaceous adenomas contain a significant percentage of mature, lipid-rich sebaceous cells which accumulate within centric segment of the neoplasm, thus highlighting categorical sebaceous differentiation and organoid configuration. Preponderant cytological atypia and an increased mitotic rate is not a feature of sebaceous adenoma and the appearance of aforesaid manifestations necessitates a re-evaluation of an essentially benign neoplasm $[3,4]$. Sebaceous adenoma depicts three distinct histologic variants such as solid, cystic and keratoacanthoma-like. Sebaceous epithelioma is a lesion which exhibits identical architectural features as sebaceous adenoma. However, undifferentiated basaloid cells quantifiably exceed the differentiated, lipidrich sebaceous cells in lobular proliferations of sebaceous adenoma. Sebaceous carcinoma also demonstrates a proliferation of basaloid cells and sebaceous cells with a solid sheet like arrangement along with features of malignancy such as mitotic figures, nuclear hyperplasia and hyperchromasia, necrosis, cellular and nuclear pleomorphism and occasional pagetoid manner of tumour dissemination (Figures 1-12) $[5,6]$.

\section{Immune Histochemical Elucidation}

Sebaceous differentiation can be validated with microscopic examination of paraffin embedded sections routinely stained with haematoxylin and eosin. However, specific

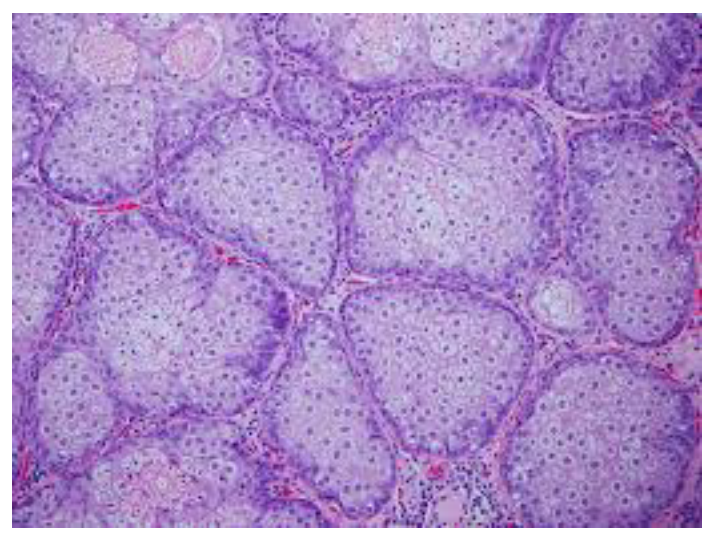

Figure 1. Sebaceous adenoma with a lobular configuration and aggregates of lipid filled sebocytes [12].

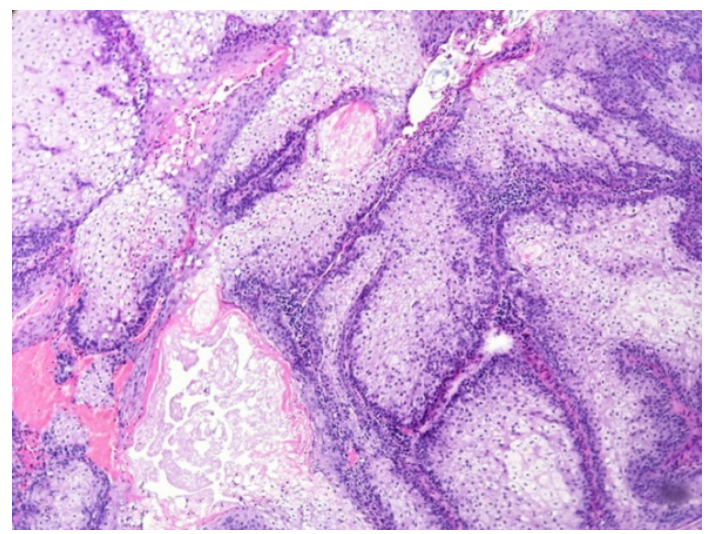

Figure 2. Sebaceous adenoma with lobules of adipose tissue and prominent circumscribing vasculature [13].

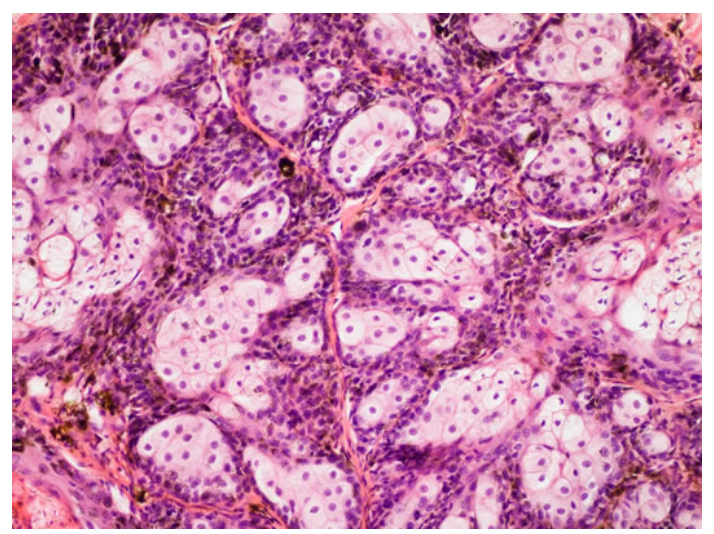

Figure 3. Sebaceous adenoma with a distinctive organoid pattern and lobules of sebum filled sebocytes [14].

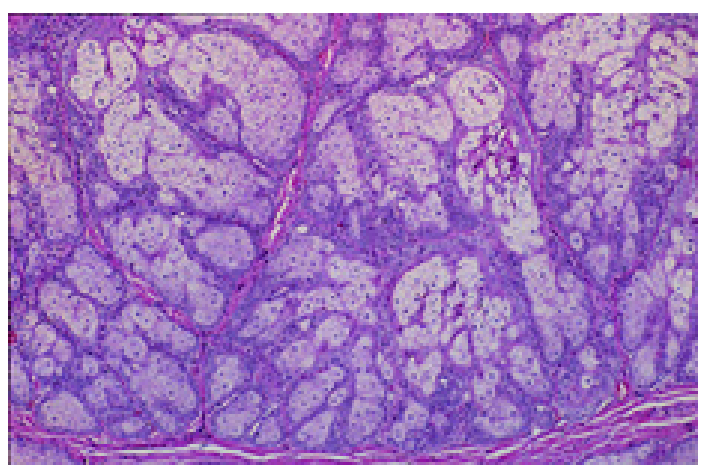

Figure 4. Sebaceous adenoma with adipose tissue in lobulated arrangement, prominently organoid intervening stroma and enhanced basaloid cells [15]. 


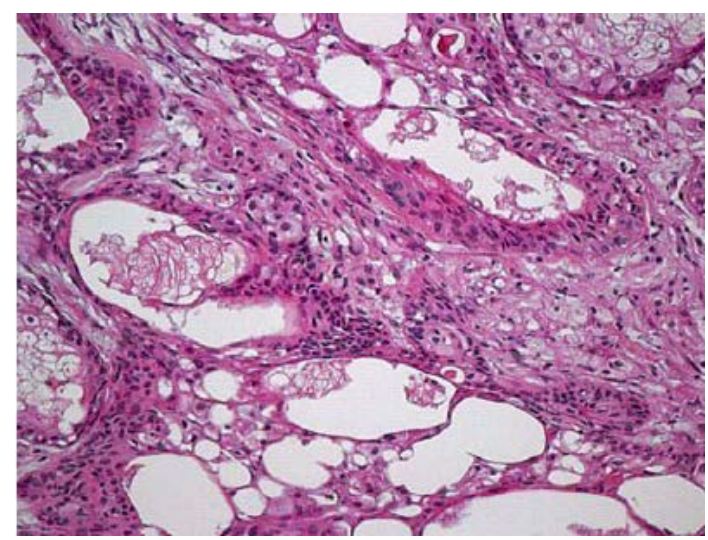

Figure 5. Sebaceous adenoma with preponderant basaloid cells and lobules of adipose tissue in a vascular architecture [16].

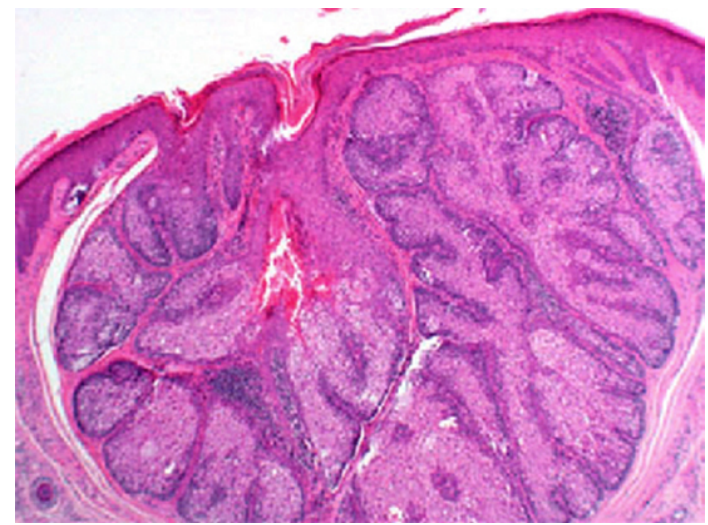

Figure 6. Sebaceous adenoma with a characteristic lobulated and organoid configuration, expansive and numerous basaloid cells and a superimposed squamous epithelium [17].

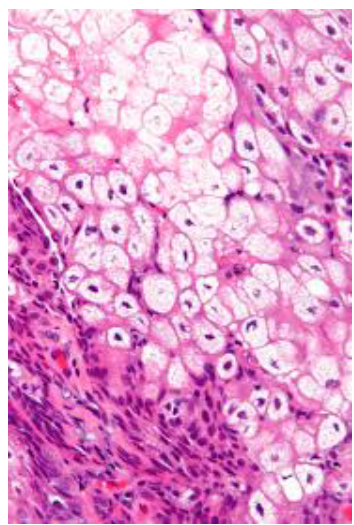

Figure 7. Sebaceous adenoma with lipid filled adipocytes and expansive basaloid cells [18].

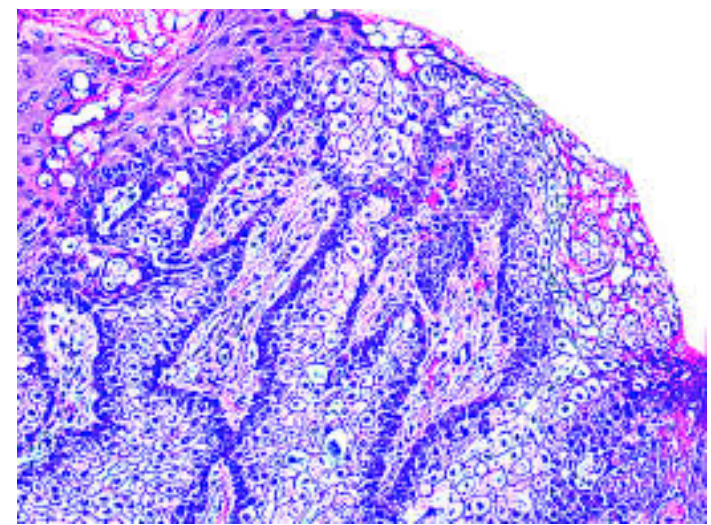

Figure 8. Sebaceous adenoma with accumulated and innumerable basaloid cells, lobules of sebocytes and organoid articulations [19].

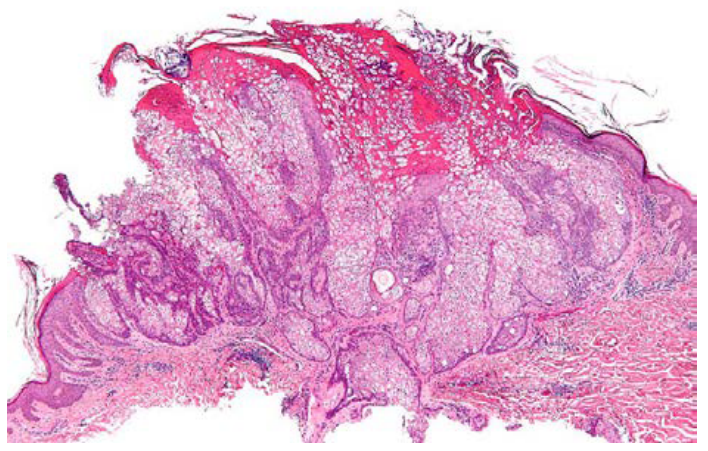

Figure 9. Sebaceous adenoma with lobular and vascular architecture with basaloid cells at the lobular perimeter [19].

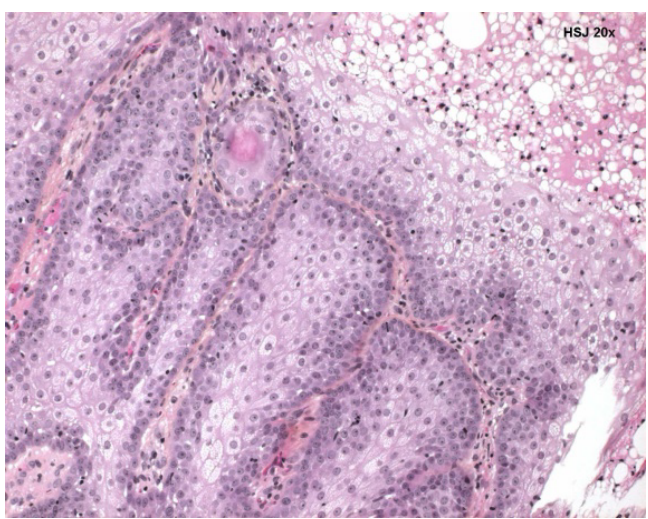

Figure 10. Sebaceous adenoma with lobules of lipid laden adipocytes, extensive marginal basaloid cells and an organoid pattern [20].

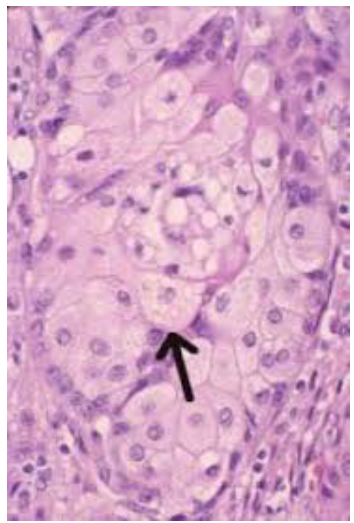

Figure 11. Sebaceous adenoma with basalold cell prominence and lipid rich adipocytes [21].

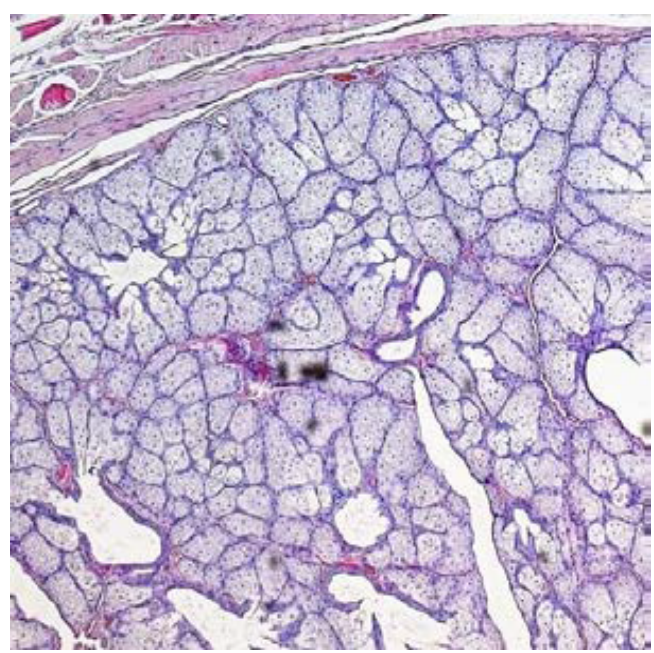

Figure 12. Sebaceous adenoma with lobules of adipocytes, basaloid cell prominence and organoid articulations [22]. 
circumstances can indicate the employment of contingent and particular immune reactions. Mature sebaceous cells are immune reactive for Epithelial membrane antigen (EMA) and non-reactive for Carcino-embryonic antigen (CEA). Basaloid cells of sebaceous neoplasm are immune reactive for cytokeratin 7 (CK7) [1,2]. Intracytoplasmic lipid content of sebaceous neoplasm can be discerned by staining with Oil Red $\mathrm{O}$ which is applicable on frozen tissue sections rather than with standard histological processing, as the aforesaid manoeuvers eliminate the cellular lipid content. Oil Red $\mathrm{O}$ stain can be appropriately performed on perioperative frozen sections. Adipophilin is a protein associated with lipid vesicles situated within the sebaceous cells and can be adopted in determining lesions with sebaceous differentiation. Aforesaid immune stain is particularly efficacious in discerning poorly differentiated sebaceous carcinoma. Which is immune non-reactive for Epithelial membrane antigen (EMA) and associated epithelial markers with the requirement of exhibiting additional, specific or sebaceous immune biomarkers [5,6]. Sebaceous adenoma depicts minimal immune reaction with p53 and Ki67 (MIB1). Podoplanin (D2-40) is a biomarker which demarcates benign from malignant sebaceous lesions. Basaloid cells of sebaceomas are immune reactive for podoplanin. Additionally, podoplanin is non-reactive or focally immune reactive in majority of sebaceous carcinomas, although basaloid variant of sebaceous carcinomas can exhibit the aforementioned immune reactivity $[7,8]$.

\section{Differential Diagnosis}

Segregation of sebaceous adenoma on histological grounds is required from lesions demonstrating a clear cytoplasm on routine haematoxylin and eosin staining such as clear cell variations of eccrine, melanocytic, keratinocytic and xanthomatous conditions or adjunctive lesions with sebaceous differentiation. Metastatic tumefaction as described with renal cell carcinoma also mandates a histological demarcation. Characteristically, clear cells with multi-vacuolated cytoplasm and crenated nuclei or mature sebaceous cells in conjunction with basaloid germinative cells are discerned on histology, although immune stains can be adopted for diagnosis in challenging circumstances. Sebaceous adenoma requires distinction from conditions such as sebaceous hyperplasia, sebaceous carcinoma and adjunctive disorders with sebaceous differentiation $[8,9]$. Contingent to the association of sebaceous adenoma with the familial Muir-Torre syndrome, subjects require an evaluation for enunciation of additional syndromes of inheritance. Apart from aforesaid Muir-Torre syndrome, sebaceous adenomas exhibiting papules or nodules on the face or body require a demarcation from inherited syndromes such as Birt-Hogg-Dube syndrome, Cowden's syndrome, tuberous sclerosis, Brooke-Spiegler syndrome and Gorlin's syndrome. Segregation of basal cell carcinoma and neoplasm with sebaceous differentiation can be achieved by immune reactions of Epithelial membrane antigen (EMA) and Ber Ep4 [2,3]. Additionally, basal cell carcinoma morphologically exhibits a predominant peripheral palisading, configuration of clefts surrounding the tumour lobules and incidental sebaceous differentiation. Epithelial membrane antigen (EMA) is immune reactive in sebaceous neoplasm whereas basal cell carcinoma are immune non-reactive. Nodular variant of basal cell carcinoma are moderately or intensely reactive for Ber Ep4 whereas sebaceous tumours are nonreactive $[9,10]$.

\section{Conclusion}

Therapeutic alleviation of cutaneous manifestations of inherited syndromes associated with sebaceous adenomas or adjunctive sebaceous neoplasm can be challenging on account of innumerable lesions and concomitant cosmetic disfigurement. Sebaceous adenoma is adequately managed by a comprehensive surgical excision which results in a minimalistic tumour reoccurrence rate. Surgical excision and cryotherapy can be employed in compliant subjects. Comprehensive extirpation of the essentially benign sebaceous adenoma is advocated by conservative surgical elimination. Verbal reassurance can be adopted for managing multiple, benign lesions $[10,11]$.

\section{References}

1. Van Walbeek K. Adenoma of the sebaceous gland of the lacrimal caruncle. Opthalmologica. 1949;117(6):370.

2. Gay JT, Gross GP. Muir Torre Syndrome. Stat Pearls Publishing. 2019.

3. Shalin SC, Lyle S, Calonje E, et al. Sebaceous neoplasia and the Muir-Torre syndrome: important connections with clinical implications. Histopathology. 2010;56(1):133-47.

4. Chauhan A, Singh S. Sebaceous adenoma. M J Opth. 2018;3(1):020.

5. Takayama K, Usui Y, Ito M, et al. A case of sebaceous adenoma of the eyelid showing excessively rapid growth. Clin Opthalmol. 2013;7(1):667-70.

6. McCarthy RL, Thomas CL, Isaacs F. Multiple benign adnexal tumours: anything but benign. Australas J Dermatol. 2019;60(3):234-6.

7. Brown S, Brennan P, Rajan N. Inherited skin tumour syndromes. Clin Med (Lond). 2017;17(6):562-7.

8. Resnik KS. Classifying neoplasm with sebaceous differentiation: a reviewer's comment. Am J Dermatopathol. 2009;31(1):94-6.

9. Kazakov DV, Kutzner H, Spagnolo DV, et al. Discordant cytological and architectural features in cutaneous sebaceous neoplasm- a classification dilemma: report of 35 cases. Am J Dermatpathol. 2009;31(1):31-6.

10. Zouboulis CC, Baron JM, Böhm M, et al. Frontiers in sebaceous gland biology and pathology. Exp Dermatol. 2008;17(6):542-51.

11. Terrell S, Wetter R, Fraga G, et al. Penile sebaceous adenoma. J Am Acad Dermatol. 2007;57(2):S42-3. 
Citation: Bajaj A. Basaloid and lobulated: Sebaceous adenoma. J Med Oncl Ther. 2019;4(1):3-7.

12. Image 1 Courtesy: Our dermatology advisor.

13. Image 2 Courtesy: Webpathology.com.

14. Image 3 Courtesy: Dermapatologiaveterinaria.com.

15. Image 4 Courtesy: Penn vet courses.

16. Image 5 Courtesy: Research gate.

17. Image 6 Courtesy: Derm 101.
18. Image 7 Courtesy: Commons Wikimedia.

19. Image 8 and 9 Courtesy: Pathology outlines.

20. Image 10 Courtesy: Humpath.

21. Image 11 Courtesy: Emedicine Medscape.

22. Image 12 Courtesy: Patalogia.cm.umk.pl.

\section{*Correspondence to:}

Dr. Roland Cheofor Ngu, MD

Douala

Cameroon

Tel: +237674789905

E-mail: rolinaro@gmail.com 